\title{
Hamiltonian dynamics of gonihedric string theory
}

\author{
Alberto Molgado ${ }^{a, b}$ and Efraín Rojas ${ }^{c}$ \\ ${ }^{a}$ Facultad de Ciencias, Universidad Autónoma de San Luis Potosí Campus \\ Pedregal, Av. Parque Chapultepec 1610, Col. Privadas del Pedregal, San Luis \\ Potosí, 78217, México \\ ${ }^{b}$ Dual CP Institute of High Energy Physics, Colima, Col, 28045, México \\ ${ }^{c}$ Facultad de Física, Universidad Veracruzana, Cto. Gonzálo Aguirre Beltrán s/n, \\ Zona Universitaria, Xalapa Veracruz 91000, México \\ E-mail: alberto.molgado@uaslp.mx \\ efrojas@uv.mx
}

\begin{abstract}
We develop in a consistent manner the Ostrogradski-Hamilton framework for gonihedric string theory. The local action describing this model, being invariant under reparametrizations, depends on the modulus of the mean extrinsic curvature of the worldsheet swept out by the string, and thus we are confronted with a genuine secondorder in derivatives field theory. In our geometric approach, we consider the embedding functions as the field variables and, even though the highly non-linear dependence of the action on these variables, we are able to complete the classical analysis of the emerging constraints for which, after implementing a Dirac bracket, we are able to identify both the gauge transformations and the proper physical degrees of freedom of the model. The Ostrogradski-Hamilton framework is thus considerable robust as one may recover in a straightforward and consistent manner some existing results reported in the literature. Further, in consequence of our geometrical treatment, we are able to unambiguously recover as a by-product the Hamiltonian approach for a particular relativistic point-particle limit associated with the gonihedric string action, that is, a model linearly depending on the first Frenet-Serret curvature.
\end{abstract}




\section{Introduction}

The so-called gonihedric string model stands for a geometric extension for the Feynman path integral over random walks to an integral over random surfaces. The action functional describing this model is defined in a way that, when the worldsheet swept out by a closed string degenerates into a worldline, it must be reduced to the action for a point-like relativistic particle [1, 2, 3]. At the classical level, gonihedric string theory has also been considered as a model for tensionless strings associated with a massless spectrum of higher integer spin gauge fields [4, 5, 6, 7] whereas, at the quantum counterpart, the model describes the manner in which quantum fluctuations generate a nonzero string tension [1, 2, 3]. Further, when the gonihedric string model is formulated on a Euclidean lattice, it has been shown that it possesses a close relation to a spin system that generalizes the Ising model with ferromagnetic, antiferromagnetic and quartic interactions [8, 9, 10, 11, 12].

The underlying geometric idea behind the gonihedric action functional is that it is proportional to the linear size of a random surface and not to its area. Thus the action functional was originally defined as proportional to a "different area element" which was constructed as a combination of both the intrinsic and the extrinsic geometry of the worldsheet. However, in its present form, the action functional for the gonihedric string may be written in a compact and elegant form that is proportional to an extrinsic area element through the modulus of the mean extrinsic curvature, $K^{i}$, where $i$ stands for the number of normal vectors of the worldsheet immersed into the background spacetime. In consequence, the action exhibits its second-order derivative nature through the extrinsic curvature definition. Although the field equations resemble wave-like equations for a unit vector, $\widehat{K}^{i}$, in the normal frame of the theory, this apparent advantage may be deceptive since the $\widehat{K}^{i}$ are non-fundamental objects which are constructed from the embedding functions $X^{\mu}$. In terms of these embedding functions, the field equations are fourth-order. In this regard, a manifestly covariant analysis on the stability for this class of higher-order in derivatives theories was recently performed [13]. Nevertheless, to the best of our knowledge, a thorough Hamiltonian analysis for this theory has been largely overlooked, mainly due to the technical difficulties involved in dealing with the canonical constraint structure and the gauge symmetries associated with the gonihedric string model.

Thus, our main aim in this paper is to develop a complete Hamiltonian analysis of the gonihedric string theory. Our approach has the advantage of bringing to the forefront the geometrical content of the phase space associated to the model. In this spirit, we borrow the existent Hamiltonian formulation for relativistic extended objects [14] which, in turn, was inspired by the Hamiltonian formulation of general relativity. This last formulation allows us to exploit in a great extent the geometry of the worldsheet in order to express the phase space quantities in terms of a reliable orthonormal 
basis. This fact simplifies and makes more evident the role that the Hamiltonian constraints play within the canonical structure. In particular, whenever we consider local actions describing extended objects of arbitrary dimension $p$ by means of the invariants that characterize the geometry of the $(p+1)$-dimensional worldvolume, at least three essential properties are mandatory: invariance under diffeomorphisms of the background spacetime, invariance under reparametrizations of the worldvolume and, when $p+1<N$, invariance under rotations of the normal vectors adapted to the worldvolume [16, 15]. For the gonihedric string theory, by choosing the embedding functions as the fundamental field variables of the theory, we are able to manage in a geometric manner the constraint structure of the Ostrogradski-Hamiltonian framework associated with the highly non-linear second-order in derivatives Lagrangian defining the model. In particular, we are capable to efficiently characterize the constraint surface which, in turn, allows us to obtain the correct gauge transformations. Indeed, for this peculiar model the last two properties give rise to the first- and the second-class constraints at the Hamiltonian level, causing that the counting of the physical degrees of freedom strongly differ from what one would expect for usual extended objects theories [14. Also, as a by-product of our geometric formulation, we discuss the point-particle limit for the gonihedric string action by directly comparing the higher-dimensional quantities to the one dimensional ones describing the relativistic curves.

Undoubtedly, once we have the Hamiltonian framework at our disposal one of the next steps to follow from our scheme is the development and understanding of the naive canonical quantization of the gonihedric model. Unfortunately, the highly non-linear dependence on the second-order derivatives of $X^{\mu}$ through the square root of $K^{i} K_{i}$, as similarly occurs in Born-Infeld-type actions, causes the canonical quantization to be rather involved. We will have occasion to explore it in its full generality. Regarding this, many interesting results have been obtained from the quantization of the model when considering a different set of independent field variables, where physical information related to the critical dimension and the massless spectrum of the model has been analyzed [6, 7].

The paper is organized as follows. In Sect. 2, we provide an overview of the gonihedric string model and emphasize the role that the extrinsic geometry plays in its description. In particular, we highligth the covariance of the model not only with respect to worldsheet diffeomorphisms but also with respect to local rotations of the normals to the worldsheet since we have a codimension of the worldsheet greater than one. In Sect. 3, using the Ostrogradski-Hamilton scheme we study the canonical structure of the theory. We show that the Hamilton equations reproduce the correct equations of motion. In Sect. 4, we develop the analysis of the constraints of the theory and make the counting of the physical degrees of freedom. We also analyse the infinitesimal canonical transformations associated to the constraints of 
the model. In Sect. 5, we explore the point-particle limit for the gonihedric string action, emphasizing the way in which this limit may be achieved within our general description. We include some concluding remarks in Sect. 6 with some comments of the work as well as a brief discussion on the drawbacks in constructing the naive canonical quantization that could arise from our Hamiltonian approach. Finally, we collect some relevant calculus related to the Poisson constraint algebra and to the functional derivatives of the constraints in a couple of appendices.

\section{The gonihedric string model}

We start with the kinematic description of the gonihedric string, [1, 2, 3, 4, 15, 7, 13] $\Sigma$, described by the action functiona $\left.\right|^{1}$

$$
S\left[X^{\mu}\right]=\alpha \int_{m} d^{2} x \sqrt{-g} \sqrt{K^{i} K_{i}},
$$

where $X^{\mu}$ denote the embedding functions which are to be taken as the fundamental field variables describing the orientable worldsheet $m$ swept out by a closed string in its evolution in an $N$-dimensional flat Minkowski spacetime, $\mathcal{M}$, with metric $\eta_{\mu \nu}=$ $\operatorname{diag}(-1,1,1, \ldots, 1)$. Lowercase Greek and lowercase Latin indices label the degrees of freedom in the background spacetime $\mathcal{M}$ and on the worldsheet $m$, respectively, thus $\mu=0,1, \ldots, N-1$ and $a=0,1$. In order to specify the string trajectory we set $x^{\mu}=X^{\mu}\left(x^{a}\right)$, where $x^{\mu}$ are local coordinates in the background spacetime $\mathcal{M}$ and $x^{a}$ are coordinates on the worldsheet $m$. Also, in action (1), $K^{i}$ stands for the trace of the $i$-th extrinsic curvature $(i, j=1,2, \ldots, N-2)$. Further, the vectors $e^{\mu}{ }_{a}:=\partial_{a} X^{\mu}$ form a basis of tangent vectors to the worldsheet $m$ which allow us to define an induced metric on $m$ as $g_{a b}:=e_{a} \cdot e_{b}$. Thus $g=\operatorname{det}\left(g_{a b}\right)$ stands for the determinant of the induced metric on the worldsheet $m$. Finally, $\alpha$ is a proportionality constant that measures the length of the surface which corresponds to the linear size of the surface, in analogy to the definition of the standard Feynman path integral [1, 2, 3]. In this sense, $[\alpha]=L^{-1}$, in Planck units.

In what follows, $\nabla_{a}$ will denote the (torsionless) covariant derivative compatible with $g_{a b}$. Similarly, the $i$-th normal vector to $m, n^{\mu i}$, is defined by the relations $e_{a} \cdot n^{i}=0$ and $n^{i} \cdot n^{j}=\delta^{i j}$, where $\delta^{i j}$ is the Kronecker delta. It is worth noting that these relations determine the normal vectors $n^{\mu i}$ up to an $O(N-2)$ rotation (and a sign) and also that they transform as a vector under normal frame rotations [17, 18].

\footnotetext{
${ }^{1}$ The action can be represented in the form $S\left[X^{\mu}\right]=\alpha \int_{m} d^{2} x \sqrt{-g} \sqrt{\Delta X \cdot \Delta X}$, where $\Delta X^{\mu}=$ $(1 / \sqrt{-g}) \partial_{a}\left(\sqrt{-g} g^{a b} \partial_{b} X^{\mu}\right)$ (see notation below). Here, the second-order nature of the field theory is explicitly written. Also note, where appropriate hereinafter, a central dot will denote contraction with respect to the Minkowski metric.
} 
Also note that whereas the tangential indices are lowered and raised with $g_{a b}$ and $g^{a b}$, respectively, the normal indices are lowered and raised with $\delta_{i j}$ and $\delta^{i j}$, respectively.

The gradients of the orthonormal basis entail the definition of the extrinsic curvature tensor, $K_{a b}^{i}:=-n^{i} \cdot \nabla_{a} \nabla_{b} X=K_{b a}^{i}$. Apart from the extrinsic curvature, whenever the codimension is higher than one, the extrinsic geometry of a surface must be complemented with the extrinsic twist potential, $\omega_{a}^{i j}$, defined by

$$
\omega_{a}^{i j}:=\nabla_{a} n^{i} \cdot n^{j}=-\omega_{a}^{j i} .
$$

In the case of a hypersurface embedding, the indice $i$ only takes one value and thus the extrinsic twist vanishes identically. Under a rotation $n^{i} \rightarrow O^{i}{ }_{j} n^{j}$, this potential transforms as a connection so that, this quantity is considered as the gauge field associated with the normal frame rotation group [19]. Therefore, to implement normal frame covariance in a manifest way, we need the existence of a new covariant derivative $\widetilde{\nabla}_{a}$, defined on fields transforming as tensors under normal frame rotations [17, 18, 19, $\widetilde{\nabla}_{a} \Phi_{j}^{i}:=\nabla_{a} \Phi_{j}^{i}-\omega_{a}^{i k} \Phi_{k j}-\omega_{a j k} \Phi^{i k}$.

The variation of the action (1) is [13]

$$
\delta S=\alpha \int_{m} d^{2} x\left(\delta(\sqrt{-g}) \sqrt{K^{i} K_{i}}+\sqrt{-g} \delta\left(\sqrt{K^{i} K_{i}}\right)\right) .
$$

The infinitesimal changes of the field variables, $X^{\mu}\left(x^{a}\right) \rightarrow X^{\mu}\left(x^{a}\right)+\delta X^{\mu}\left(x^{a}\right)$, may be decomposed into tangential and normal deformations, that is, $\delta X^{\mu}=\Phi^{a} e^{\mu}{ }_{a}+\Phi^{i} n^{\mu}{ }_{i}$ where $\Phi^{a}$ and $\Phi^{i}$ denote both tangential and normal deformation fields, respectively. On the other hand, as the action (1) is invariant under reparametrizations of the worldsheet, this gauge symmetry determines that only the transverse worldsheet motion is physical and, in consequence, the tangential deformations, $\delta_{\|} X^{\mu}=\Phi^{a} \partial_{a} X^{\mu}$, are usually ignored [20]. Therefore, it is only necessary to consider the normal deformations $\delta_{\perp} X^{\mu}=\Phi^{i} n^{\mu}{ }_{i}$, where the $\Phi^{i}$ are assumed to be functions of $x^{a}$. Consequently, following the development performed in [20, 13], the variation (3) becomes

$$
\delta_{\perp} S=-\alpha \int_{m} d^{2} x \sqrt{-g}\left(\widetilde{\Delta} \widehat{K}^{i}-J_{j}^{a b} K_{a b}^{i} \widehat{K}^{j}\right) \Phi_{i},
$$

up to a total derivative term. Here the symbol $\widetilde{\Delta}=g^{a b} \widetilde{\nabla}_{a} \widetilde{\nabla}_{b}$ denotes the worldsheet d'Alembertian operator and, $J_{i}^{a b}:=g^{a b} K_{i}-K_{i}^{a b}$ is a tensor, and

$$
\widehat{K}^{i}:=\frac{K^{i}}{\sqrt{K^{j} K_{j}}}
$$

is a unit vector which may be thought of as defining the coordinates of a $S^{(N-2)}$ unit sphere, $\delta_{i j} \widehat{K}^{i} \widehat{K}^{j}=1$. Evidently, $\widehat{K}^{i}$ transforms like a vector under normal frame rotations. The tensor $J_{i}^{a b}$ is conserved in the sense that $\widetilde{\nabla}_{a} J_{i}^{a b}=0$ which stands as 
a consequence of the Codazzi-Mainardi integrability condition for surfaces immersed in a background spacetime is Minkowski [21. Hence, the classical string trajectories are obtained from the $N-2$ field equations

$$
\left(\widetilde{\Delta} \delta_{j}^{i}-J_{j}^{a b} K_{a b}^{i}\right) \widehat{K}^{j}=0 .
$$

On pedagogical grounds, this set of equations may be seen as a set of wave-like equations for the variables $\widehat{K}^{i}$, but we must remember that the field variables are the embedding functions. Regarding this point, the field equations (6) are fourth-order in derivatives of $X^{\mu}$, a fact that can be directly seen once we write the extrinsic curvature in terms of the derivatives of the embedding functions. The field equations (6) can also be obtained from the conserved stress tensor, $f^{\mu a}$, associated with the model. Certainly, for an extended object, the conservation laws associated with the Poincaré symmetry of the ambient spacetime can be written in terms of a conserved tensor, $f^{\mu a}$, which turns out to be the conserved linear momentum. In this sense, by projecting $\nabla_{a} f^{\mu a}=0$ along the tangential directions of $m$, we obtain the gauge redundancy through Bianchi identitites whereas the normal projection of the conservation law, $n^{i}$. $\nabla_{a} f^{a}=0$, provides the equations of motion of the theory. Guided by the variational techniques developed in [16], the conserved stress tensor is

$$
f^{\mu a}=-\alpha\left(\widehat{K}^{i} J_{i}^{a b} e_{b}^{\mu}+g^{a b} \widetilde{\nabla}_{b} \widehat{K}^{i} n^{\mu}{ }_{i}\right) .
$$

From the definition and properties of the extrinsic curvature $K_{a b}^{i}$, and the CodazziMainardi equation [20, 16], the field equations (6) follow. Note that the stress tensor (7) is written in terms of the worldsheet basis, $\left(e^{\mu}{ }_{b}, n^{\mu}{ }_{i}\right)$. For Hamiltonian purposes, a very important physical quantity can be constructed from (7) and the geometry of the string $\Sigma$ as seen from the perspective of the worldsheet $m,[14$

$$
\pi^{\mu}:=\sqrt{h} \eta_{a} f^{\mu a}=-\alpha \sqrt{h}\left(\widehat{K}^{i} J_{i}^{a b} \eta_{a} e^{\mu}{ }_{b}+\eta^{a} \widetilde{\nabla}_{a} \widehat{K}^{i} n^{\mu}{ }_{i}\right)
$$

where $\eta^{a}$ is a unit time normal vector from the string $\Sigma$ into the worldsheet $m$, and $h$ is the determinant of the spatial metric $h_{A B}$ to $\Sigma$, see below for details. Note that uppercase Latin indices here stand for the unique value that labels the only spatial coordinate, usually denoted by $\sigma$, in the conventional string theory framework. ${ }^{2}$ Indeed, (8) represents the conserved linear momentum density associated with the Poincaré invariance of the gonihedric model under background translations [16].

Owing to the rich geometrical nature of the model (1), the introduction of the unit vector (5) not only contributes to simplicity in expressing the field equations (6) but it also resulted convenient in order to construct the projector

$$
\Pi^{i j}=\delta^{i j}-\widehat{K}^{i} \widehat{K}^{j}
$$

\footnotetext{
${ }^{2}$ In fact, in the case under study here, the determinant $h$ corresponds to the unique component of the spatial metric $h_{A B}$. However, we would like to keep the notation as general as possible in order to easily adapt it to any generalization.
} 
This projector sends fields that transform as tensors under normal frame rotations onto the hyperplanes transverse to the $\widehat{K}^{i}$ vectors. Indeed, it turns out that $\Pi^{i j} \widehat{K}_{j}=$ 0 and $\Pi^{i j} \widetilde{\nabla}_{a} \widehat{K}_{j}=\widetilde{\nabla}_{a} \widehat{K}^{i}$. These facts, together with the orthogonality property $\delta_{i j} \widehat{K}^{i} \widetilde{\nabla}_{a} \widehat{K}^{j}=0$, signal that $\left\{\widetilde{\nabla}_{a} K^{i}, \widehat{K}^{i}\right\}$ span an orthonormal basis for a unit sphere $S^{(N-2)}$. Further, notice that for the case of a hypersurface, $i=1$, we have that $\Pi^{i j}$ vanishes. In consequence, we realize that we have a locally defined Gauss normal map, $\Phi: m \rightarrow S^{(N-2)}$, that maps points $p \in m$ to the unit sphere $S^{(N-2)}$, Fig. 1. Intuitively, for the present model, this Gauss map allows us to interpret the alleged invariance under rotations of the normal vectors adapted to the worldsheet, as discussed in the Introduction within the general extended objects setup [21].

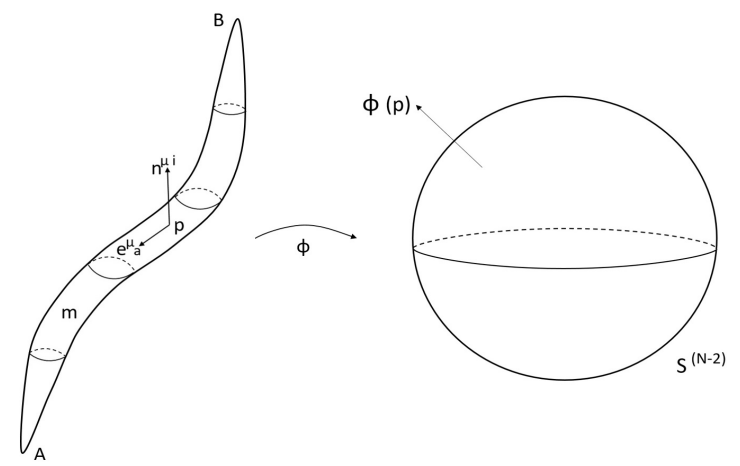

Figure 1: The Gauss map $\Phi: m \rightarrow S^{(N-2)}$, maps points $p$ defined on the worldsheet to the unit sphere $S^{(N-2)}$.

\section{Ostrogradski-Hamilton analysis}

The general aspects of the Ostrogradski-Hamilton analysis for second-order in derivatives models for extended objects have been presented in detail in [14. Within the ADM framework adapted to extended objects 22, 14, in addition to the embedding functions, $X^{\mu}$, and their velocities, $\dot{X}^{\mu}$, the action (1) is also dependent on the accelerations $\ddot{X}^{\mu}$. On the hypersurface $\Sigma_{t}$, that is, the string $\Sigma$ considered at a fixed time $t$, we have the orthonormal basis $\left\{\epsilon^{\mu}{ }_{\sigma}, \eta^{\mu}, n^{\mu i}\right\}$, where we have defined $\epsilon^{\mu}{ }_{\sigma}:=\partial_{\sigma} X^{\mu}$. The local action of the gonihedric string field theory (1) can be written as $S\left[X^{\mu}\right]=\int_{m} d^{2} x N \sqrt{h} L\left(g_{a b}, K_{a b}^{i}\right)$ with $L=\alpha \sqrt{K^{i} K_{i}}$. Here, $h$ denotes the determinant of the induced metric on $\Sigma_{t}$ denoted by $h_{A B}=\eta_{\mu \nu} \epsilon_{\sigma}^{\mu} \epsilon_{\sigma}^{\nu}$, where $\epsilon_{\sigma}^{\mu}$ is equivalent to the tangent vector $e^{\mu}{ }_{\sigma}$ at any point in the hypersurface $\Sigma_{t}$. The mean extrinsic curvature acquires the form

$$
K^{i}=\frac{1}{N^{2}}\left[\left(n^{i} \cdot \ddot{X}\right)-2 N^{\sigma}\left(n^{i} \cdot \mathcal{D}_{\sigma} \dot{X}\right)-\left(N^{2} h^{\sigma \sigma}-N^{\sigma} N^{\sigma}\right)\left(n^{i} \cdot \mathcal{D}_{\sigma} \mathcal{D}_{\sigma} X\right)\right],
$$


(see reference [14] for details). The symbols $N$ and $N^{\sigma}$ denote the lapse function and the shift vector defined on $\Sigma_{t}$, respectively, obtained in a similar way as in the ADM formulation for general relativity [23. Note that the velocities may be written in terms of the lapse function and the shift vector as $\dot{X}^{\mu}=N \eta^{\mu}+N^{\sigma} \epsilon_{\sigma}^{\mu}$. Also note that $\mathcal{D}_{\sigma}$ in $(10)$ stands for the covariant derivative such that $\mathcal{D}_{\sigma} h_{\sigma \sigma}=0$.

The momenta $p_{\mu}$ and $P_{\mu}$, conjugate to $X^{\mu}$ and $\dot{X}^{\mu}$, respectively, in terms of the worldsheet basis, are [14]

$$
\begin{aligned}
P_{\mu}:= & \frac{\partial L}{\partial \ddot{X}^{\mu}}=-\frac{\sqrt{h}}{N}\left(\eta_{a} L_{i}^{a b} \eta_{b}\right) n_{\mu}{ }^{i} \\
p_{\mu}:= & \frac{\partial L}{\partial \dot{X}^{\mu}}-\frac{d}{d \tau}\left(\frac{\partial L}{\partial \ddot{X}^{\mu}}\right)=-\sqrt{h}\left[\left(L \eta^{a}-2 \eta_{b} L_{i}^{c(a} K_{c}^{b) i}\right) e_{\mu a}+\eta_{b} \nabla_{a}\left(L_{i}^{a b} n_{\mu}{ }^{i}\right)\right] \\
& +\partial_{\sigma}\left[N^{\sigma} P_{\mu}-\sqrt{h}\left(\eta_{b} L_{i}^{a b} \epsilon_{a}^{\sigma}\right) n_{\mu}{ }^{i}\right]
\end{aligned}
$$

where we have defined $L_{i}^{a b}:=\partial L / \partial K_{a b}^{i}$. In our particular case we have $L_{i}^{a b}=\alpha \widehat{K}_{i} g^{a b}$. Thus, by considering the Gauss-Weingarten equation $\widetilde{\nabla}_{a} n^{\mu i}=K_{a}{ }^{b i} e^{\mu}{ }_{b}$, the momenta reduces to

$$
\begin{aligned}
& P_{\mu}=\alpha \frac{\sqrt{h}}{N} \widehat{K}^{i} n_{\mu i}, \\
& p_{\mu}=\pi_{\mu}+\partial_{\sigma}\left(N^{\sigma} P_{\mu}\right),
\end{aligned}
$$

where $\pi^{\mu}$ is defined in (8) and we have considered the identity $g_{a b} \eta^{a} \epsilon_{\sigma}^{b}=0$. We have then an $8 N$-dimensional phase space, $\Gamma$, spanned by $\left\{X^{\mu}, p_{\mu} ; \dot{X}^{\mu}, P_{\mu}\right\}$. It should be stressed that within this ADM approach for extended objects the boundary term $\partial_{\sigma}\left(N^{\sigma} P_{\mu}\right)=\mathcal{L}_{\vec{N}} P_{\mu}$ is always present [14]. In this extended phase space, the corresponding Legendre transformation $\mathcal{H}_{0}:=p \cdot \dot{X}+P \cdot \ddot{X}-\mathcal{L}$ with $\mathcal{L}=\alpha N \sqrt{h} \sqrt{K^{i} K_{i}}$, provides the canonical Hamiltonian

$$
\mathcal{H}_{0}=p \cdot \dot{X}+2 N^{\sigma}\left(P \cdot \mathcal{D}_{\sigma} \dot{X}\right)+\left(N^{2} h^{\sigma \sigma}-N^{\sigma} N^{\sigma}\right)\left(P \cdot \mathcal{D}_{\sigma} \mathcal{D}_{\sigma} X\right) .
$$

We would like to emphasize the linear dependence of the canonical Hamiltonian on the momenta $p_{\mu}$ and $P_{\mu}$. Classically, these momenta can take both negative and positive values in phase space making the canonical Hamiltonian unbounded from below, thus reaching both positive and negative values. In other words, Ostrogradski instabilities may be present in the dynamics of the model. Additionally, $\mathcal{H}_{0}$ contains a highly non-linear dependence on the configuration variables $X^{\mu}$ and $\dot{X}^{\mu}$ by virtue of the lapse function, the shift vector and the induced metric.

For the model of our interest, the presence of symmetries manifests trough the presence of constraints. We can determine these symmetries by computing first the null eigenvectors of the Hessian matrix

$$
\mathcal{H}_{\mu \nu}:=\frac{\partial^{2} \mathcal{L}}{\partial \ddot{X}^{\mu} \partial \ddot{X}^{\nu}}=\alpha \frac{\sqrt{h}}{N^{3} \sqrt{K^{l} K_{l}}} \Pi^{i j} n_{\mu i} n_{\nu j},
$$


where $\Pi^{i j}$ stands for the projector defined in $(9)$. One can easily be convinced that, due to the orthonormal property of the $\Sigma_{t}$ basis, the eigenvectors with zero eigenvalues of $\mathcal{H}_{\mu \nu}$ are given by $\dot{X}^{\mu}, \epsilon_{\sigma}^{\mu}$ and $P_{\mu}$. Guided by the canonical formalism developed for second-order singular theories [24, 14], by projecting the momenta (13) along these zero-modes we get three primary constraints

$$
\begin{aligned}
C_{1} & =P \cdot \dot{X}=0 \\
C_{\sigma} & =P \cdot \partial_{\sigma} X=0, \\
C_{2} & =N^{2} P^{2}-\alpha^{2} h=0 .
\end{aligned}
$$

It is convenient to turn these constraints densities into functions in $\Gamma$. Hence, we must smear out $C_{1}, C_{\sigma}$ and $C_{2}$ by test fields $\lambda, \lambda^{\sigma}$ and $\phi$ on $\Sigma_{t}$

$$
\begin{aligned}
\mathcal{S}_{\lambda} & =\int_{\Sigma_{t}} d \sigma \lambda P \cdot \dot{X} \\
\mathcal{V}_{\vec{\lambda}} & =\int_{\Sigma_{t}} d \sigma \lambda^{\sigma} P \cdot \partial_{\sigma} X \\
\mathcal{W}_{\phi} & =\int_{\Sigma_{t}} d \sigma \phi\left(N^{2} P^{2}-\alpha^{2} h\right) .
\end{aligned}
$$

For any two phase space functions, $F, G \in \Gamma$, the corresponding Poisson bracket $(\mathrm{PB})$ is defined by

$$
\{F, G\}=\int_{\Sigma_{t}} d \sigma\left[\frac{\delta F}{\delta X^{\mu}} \frac{\delta G}{\delta p_{\mu}}+\frac{\delta F}{\delta \dot{X}^{\mu}} \frac{\delta G}{\delta P_{\mu}}-\frac{\delta F}{\delta p_{\mu}} \frac{\delta G}{\delta X^{\mu}}-\frac{\delta F}{\delta \dot{X}^{\mu}} \frac{\delta G}{\delta P_{\mu}}\right] .
$$

Thus, under the PB the primary constraints functions 20, 21 and (22) are in involution

$$
\begin{array}{ll}
\left\{\mathcal{S}_{\lambda}, \mathcal{S}_{\lambda^{\prime}}\right\}=0, & \left\{\mathcal{V}_{\vec{\lambda}}, \mathcal{V}_{\overrightarrow{\lambda^{\prime}}}\right\}=0, \\
\left\{\mathcal{S}_{\lambda}, \mathcal{V}_{\vec{\lambda}}\right\}=\mathcal{V}_{\overrightarrow{\lambda^{\prime}}} \quad \lambda^{\prime} \sigma=\lambda \lambda^{\sigma}, & \left\{\mathcal{V}_{\vec{\lambda}}, \mathcal{W}_{\phi}\right\}=0, \\
\left\{\mathcal{S}_{\lambda}, \mathcal{W}_{\phi}\right\}=0, & \left\{\mathcal{W}_{\phi}, \mathcal{W}_{\phi^{\prime}}\right\}=0
\end{array}
$$

Therefore, we must have at least three secondary constraints. Following the DiracBergmann procedure for constrained systems, this can be proved by considering the temporal evolution of the primary constraints generated by the total Hamiltonian

$$
H_{T}=H_{0}+\mathcal{S}_{\lambda}+\mathcal{V}_{\vec{\lambda}}+\mathcal{W}_{\phi}
$$

where $H_{0}:=\int_{\Sigma_{t}} d \sigma \mathcal{H}_{0}$. However, bearing in mind conditions 24 , we will follow a more convenient strategy to obtain the secondary constraints which consists on projecting the momenta (14) along the null eigenvectors of the Hessian matrix (16), as discussed in detail in [24], and thus we readily obtain

$$
C_{3}=p \cdot \dot{X}+2 N^{\sigma} P \cdot \mathcal{D}_{\sigma} \dot{X}+\left(N^{2} h^{\sigma \sigma}-N^{\sigma} N^{\sigma}\right) P \cdot \mathcal{D}_{\sigma} \mathcal{D}_{\sigma} X=0
$$




$$
\begin{aligned}
& \mathcal{C}_{\sigma}=p \cdot \partial_{\sigma} X+P \cdot \partial_{\sigma} \dot{X}=0, \\
& C_{4}=p \cdot P-P \cdot \partial_{\sigma}\left(N^{\sigma} P\right)=0,
\end{aligned}
$$

where we have used that $\pi \cdot P=0$ which can be proved from the fact that $\widehat{K}_{i} \widetilde{\nabla}_{a} \widehat{K}^{i}=0$. As before, we can get constraint functions in phase space by smearing out $C_{3}, \mathcal{C}_{\sigma}$ and $C_{4}$ by the test fields $\Lambda, \Lambda^{\sigma}$ and $\Phi$ defined on $\Sigma_{t}$

$$
\begin{aligned}
S_{\Lambda} & =\int_{\Sigma_{t}} d \sigma \Lambda\left[p \cdot \dot{X}+2 N^{\sigma} P \cdot \mathcal{D}_{\sigma} \dot{X}+\left(N^{2} h^{\sigma \sigma}-N^{\sigma} N^{\sigma}\right) P \cdot \mathcal{D}_{\sigma} \mathcal{D}_{\sigma} X\right], \\
V_{\vec{\Lambda}} & =\int_{\Sigma_{t}} d \sigma \Lambda^{\sigma}\left(p \cdot \partial_{\sigma} X+P \cdot \partial_{\sigma} \dot{X}\right), \\
W_{\Phi} & =\int_{\Sigma_{t}} d \sigma \phi\left[p \cdot P-P \cdot \partial_{\sigma}\left(N^{\sigma} P\right)\right] .
\end{aligned}
$$

When evolving in time the secondary constraints by using the results $\left\{S_{\Lambda}, H_{T}\right\} \approx$ $0,\left\{V_{\vec{\Lambda}}, H_{T}\right\} \approx 0$ and $\left\{W_{\Phi}, H_{T}\right\} \approx 0$ and considering the PB listed in Section 7 , we do not obtain tertiary constraints, except for the point-particle limit to be discussed later in Section 5, so the program of generating further constraints is therefore finished.

The constraints (17), (18) are characteristic of second-order in derivatives brane models [14]. Note that these constraints just involve the momenta $P_{\mu}$, and geometrically they may be interpreted as a consequence of the orthonormality of the worldsheet basis. On the contrary, the constraints $(26)$ and (27) involve all the phase space variables. On the one hand, (26) reflects the vanishing of the canonical Hamiltonian which is expected because of the invariance under reparametrizations of the theory. Certainly, it generates diffeomorphisms out of $\Sigma_{t}$ onto the worldsheet. On the other hand, $\mathcal{C}_{\sigma}=0$, generates diffeomorphisms tangential to $\Sigma_{t}$. This can be verified by considering the Poisson brackets with the phase space variables as we will discuss later. Regarding the constraints $(19)$ and (28), the first only reflects the space-like nature of the highest momenta $P_{\mu}$, whereas the latter reflects the orthogonality between the momenta $\pi_{\mu}$ and $P_{\mu}$. From the geometrical point of view and regarding the Gauss map, we may depict the momenta $\pi_{\mu}$ and $P_{\mu}$ as vectors tangent and normal to the $S^{(N-2)}$ sphere, respectively. In fact, $(19)$ and $(28)$ will be characterized as second-class constraints in Dirac's terminology, as we will discuss below.

\subsection{Hamilton equations}

In this section, we will obtain the field equations from the Hamiltonian point of view. In particular, the Hamiltonian equations will be helpful in order to fix the Lagrange multipliers $\lambda, \lambda^{A}$ and $\phi$ in terms of the canonical variables. By using the functional derivatives listed in Section 8 we have first

$$
\partial_{t} X^{\mu}=\frac{\delta H_{T}}{\delta p_{\mu}}=\frac{\delta H_{0}}{\delta p_{\mu}}=\dot{X}^{\mu},
$$


which is a trivial identity since the only dependence on $p_{\mu}$ is trough the term $p \cdot \dot{X}$ appearing in $H_{0}$. Second, we have

$$
\begin{aligned}
\partial_{t} \dot{X}^{\mu} & =\frac{\delta H_{T}}{\delta P_{\mu}}=\frac{\delta H_{0}}{\delta P_{\mu}}+\frac{\delta \mathcal{S}_{\lambda}}{\delta P_{\mu}}+\frac{\delta \mathcal{V}_{\vec{\lambda}}}{\delta P_{\mu}}+\frac{\delta \mathcal{W}_{\phi}}{\delta P_{\mu}} \\
& =\left(N^{2} h^{\sigma \sigma}-N^{\sigma} N^{\sigma}\right) \mathcal{D}_{\sigma} \mathcal{D}_{\sigma} X^{\mu}+2 N^{\sigma} \mathcal{D}_{\sigma} \dot{X}^{\mu}+\lambda \dot{X}^{\mu}+\lambda^{\sigma} \epsilon_{\sigma}^{\mu} \\
& +2 \phi N^{2} P^{\mu}
\end{aligned}
$$

At this stage we are able to unambiguously fix the Lagrange multipliers. Indeed, by contracting (33) with the momenta $P_{\mu}$, and by considering the constraint (19) we obtain

$$
\phi=\frac{N}{2 \alpha \sqrt{h}} \sqrt{K^{i} K_{i}} .
$$

Here, we have used the general expression for the acceleration of an extended object (see equation (106)). Now, contracting (33) with $\eta^{\mu}$ and $\epsilon_{\sigma}^{\mu}$, respectively, and considering the constraints (17) and $(18)$ we have

$$
\begin{aligned}
\lambda & =\mathcal{D}_{\sigma} N^{\sigma}-\frac{N^{2}}{\sqrt{h}} \eta^{a} \nabla_{a}\left(\frac{\sqrt{h}}{N}\right) \\
\lambda^{\sigma} & =N h^{\sigma \sigma} \mathcal{D}_{\sigma} N-N^{\sigma} \mathcal{D}_{\sigma} N^{\sigma}+\frac{N^{2}}{\sqrt{h}} \eta^{a} \nabla_{a}\left(\sqrt{h} \frac{N^{\sigma}}{N}\right),
\end{aligned}
$$

where we have used the time derivatives of the spatial metric $h_{\sigma \sigma}$ and its determinant (see formulae (107) and (108), respectively). Next, by considering the temporal evolution of the momenta $P^{\mu}$, we see that the Hamilton equations only reproduces the definition of the momenta $p_{\mu}$,

$$
\begin{aligned}
\partial_{t} P_{\mu} & =-\frac{\delta H_{T}}{\delta \dot{X}^{\mu}}=-\frac{\delta H_{0}}{\delta \dot{X}^{\mu}}-\frac{\delta \mathcal{S}_{\lambda}}{\delta \dot{X}^{\mu}}-\frac{\delta \mathcal{V}_{\vec{\lambda}}}{\delta \dot{X}^{\mu}}-\frac{\delta \mathcal{W}_{\phi}}{\delta \dot{X}^{\mu}}, \\
& =-p_{\mu}+2 N\left(P \cdot \mathcal{D}_{\sigma} \mathcal{D}^{\sigma} X\right) \eta_{\mu}+2 N^{\sigma}\left(P \cdot \mathcal{D}_{\sigma} \mathcal{D}_{\sigma} X\right) \epsilon_{\mu}{ }^{\sigma}-2\left(P \cdot \mathcal{D}_{\sigma} \dot{X}\right) \epsilon_{\mu}{ }^{\sigma} \\
& +\mathcal{D}_{\sigma}\left(2 N^{\sigma} P_{\mu}\right)-\lambda P_{\mu}+2 \phi N P^{2} \eta_{\mu} .
\end{aligned}
$$

Inserting the Lagrange multipliers $(34)$ and 36 into this expression leads to

$$
\begin{aligned}
p_{\mu} & =\alpha \sqrt{h} \widehat{K}_{i} K^{i} \eta_{\mu}+2 N h^{\sigma \sigma}\left(P \cdot \mathcal{D}_{\sigma} \mathcal{D}_{\sigma} X\right) \eta_{\mu}+2 N^{\sigma}\left(P \cdot \mathcal{D}_{\sigma} \mathcal{D}_{\sigma} X\right) \epsilon_{\mu}{ }^{\sigma} \\
& -2\left(P \cdot \mathcal{D}_{\sigma} \dot{X}\right) \epsilon_{\mu}{ }^{\sigma}-\alpha \sqrt{h} \eta^{a} \nabla_{a}\left(\widehat{K}_{i} n_{\mu}{ }^{i}\right)+\partial_{\sigma}\left(N^{\sigma} P_{\mu}\right),
\end{aligned}
$$

where we have considered the fact that $\partial_{t} P_{\mu}=\dot{X}^{a} \nabla_{a} P_{\mu}=N \eta^{a} \nabla_{a} P_{\mu}+N^{\sigma} \epsilon^{a}{ }_{\sigma} \nabla_{a} P_{\mu}$. This expression matches (12) or (14) once we write (8) in terms of the phase space variables. Finally, the fourth set of Hamilton equations

$$
\partial_{t} p_{\mu}=-\frac{\delta H_{T}}{\delta X^{\mu}}=-\frac{\delta H_{0}}{\delta X^{\mu}}-\frac{\delta \mathcal{V}_{\vec{\lambda}}}{\delta X^{\mu}}-\frac{\delta \mathcal{W}_{\phi}}{\delta X^{\mu}},
$$

represents the field equations of motion (6) in its canonical form as it may be straightforwardly seen by introducing the Lagrange multipliers (34) and (35). 


\section{First- and second-class constraints}

Next, with the intention of characterizing the constraint surface, we will separate both the primary and secondary constraints into first- and second-class constraints. To perform this we construct the antisymmetric matrix composed of the PB of all the constraint functions ${ }^{3}, \Omega_{a b}:=\left\{\mathfrak{C}_{a}, \mathfrak{C}_{b}\right\}$, where we have chosen the following order

$$
\mathfrak{C}_{a}=\left\{\mathcal{S}_{\lambda}, \mathcal{V}_{\vec{\lambda}}, \mathcal{W}_{\phi}, S_{\Lambda}, V_{\vec{\Lambda}}, W_{\Phi}\right\}, \quad a, b=1,2, \ldots, 6 .
$$

The matrix $\Omega_{a b}$ weakly reads

$$
\left(\Omega_{a b}\right) \approx\left(\begin{array}{cccccc}
0 & 0 & 0 & 0 & 0 & 0 \\
0 & 0 & 0 & 0 & 0 & 0 \\
0 & 0 & 0 & 0 & 0 & \mathbb{A} \\
0 & 0 & 0 & 0 & 0 & \mathbb{B} \\
0 & 0 & 0 & 0 & 0 & \mathbb{C} \\
0 & 0 & -\mathbb{A} & \mathbb{B} & -\mathbb{C} & \mathbb{F}
\end{array}\right),
$$

where the non-vanishing entries, $\mathbb{A}, \mathbb{B}, \mathbb{C}$ and $\mathbb{F}$, are defined in Subsection 7.1. The rank of this matrix is 2, thus pointing out the existence of two second-class constraints. In order to select these second-class constraints we need to determine first the four zero eigenvectors $u_{(I)}^{a}$, such that $\Omega_{a b} u_{(I)}^{b}=0$, with $I=1,2,3,4$. These vectors can be taken as

$$
\begin{aligned}
& \vec{u}_{(1)}=(1,0,0,0,0,0), \\
& \vec{u}_{(2)}=(0,1,0,0,0,0), \\
& \vec{u}_{(3)}=(0,0,-\mathbb{B} / \mathbb{A}, 1,0,0), \\
& \vec{u}_{(4)}=(0,0,-\mathbb{C} / \mathbb{A}, 0,1,0) .
\end{aligned}
$$

It is straightforward to see that the functions $\mathcal{F}_{I}:=u_{(I)}^{a} \mathfrak{C}_{a}$ are first-class constraints. Explicitly,

$$
\begin{aligned}
\mathcal{F}_{1} & =\mathfrak{C}_{1}=\mathcal{S}_{\lambda}, \\
\mathcal{F}_{2} & =\mathfrak{C}_{2}=\mathcal{V}_{\vec{\lambda}}, \\
\mathcal{F}_{3} & =\mathfrak{C}_{4}-\frac{\mathbb{B}}{\mathbb{A}} \mathfrak{C}_{3}=S_{\Lambda}-\frac{\mathbb{B}}{\mathbb{A}} \mathcal{W}_{\phi}, \\
\mathcal{F}_{4} & =\mathfrak{C}_{5}-\frac{\mathbb{C}}{\mathbb{A}} \mathfrak{C}_{3}=V_{\vec{\Lambda}}-\frac{\mathbb{C}}{\mathbb{A}} \mathcal{W}_{\phi} .
\end{aligned}
$$

In this spirit, if we are able to choose a set of linearly independent vectors $u_{\left(I^{\prime}\right)}^{a},\left(I^{\prime}=\right.$ $5,6)$, such that they do not depend on the vectors $u_{(I)}^{a}$ and also following the condition

\footnotetext{
${ }^{3}$ In this section, the indices $a$ and $b$ should not be confused with those characterizing the worldsheet geometry.
} 
$\operatorname{det}\left(u_{\left(a^{\prime}\right)}^{a}\right) \neq 0$ with $a^{\prime}=\left(I, I^{\prime}\right)$, then the functions $S_{I^{\prime}}:=u_{\left(I^{\prime}\right)}^{a} \mathfrak{C}_{a}$ are second-class constraints. Indeed, by choosing

$$
\begin{aligned}
& \vec{u}_{5}=(0,0,1,0,0,0), \\
& \vec{u}_{6}=(0,0,0,0,0,1),
\end{aligned}
$$

then

$$
\begin{aligned}
\mathcal{S}_{5} & =\mathcal{W}_{\phi}, \\
\mathcal{S}_{6} & =W_{\Phi},
\end{aligned}
$$

are second-class constraints. Hence, the constraints $\mathcal{F}_{I}$ and $\mathcal{S}_{I^{\prime}}$ define an equivalent representation of the constrained phase space. In this new representation of the constraint surface we can introduce the matrix $\mathcal{S}_{I^{\prime} J^{\prime}}:=\left\{\mathcal{S}_{I^{\prime}}, \mathcal{S}_{J^{\prime}}\right\}, I^{\prime}, J^{\prime}=5,6$; and its inverse $\left(\mathcal{S}^{-1}\right)^{I^{\prime} J^{\prime}}$, given by

$$
\left(\mathcal{S}_{I^{\prime} J^{\prime}}\right)=\left(\begin{array}{cc}
0 & \mathbb{A} \\
-\mathbb{A} & \mathbb{F}
\end{array}\right) \quad \text { and } \quad\left(\left(\mathcal{S}^{-1}\right)^{I^{\prime} J^{\prime}}\right)=\frac{1}{\mathbb{A}^{2}}\left(\begin{array}{cc}
\mathbb{F} & -\mathbb{A} \\
\mathbb{A} & 0
\end{array}\right) \text {. }
$$

respectively. The inverse of the matrix $\left(\mathcal{S}_{I^{\prime} J^{\prime}}\right)$ allows us to introduce the Dirac bracket (DB) in the usual way

$$
\{F, G\}_{D}:=\{F, G\}-\left\{F, \mathcal{S}_{I^{\prime}}\right\}\left(\mathcal{S}^{-1}\right)^{I^{\prime} J^{\prime}}\left\{\mathcal{S}_{J^{\prime}}, G\right\}
$$

Once we have identified the set of second-class constraints, and after imposing the Dirac bracket, we can consistently set those constraints equal to zero [26], so that the first-class constraints (48) and (49) reduce to

$$
\begin{aligned}
\mathcal{F}_{3} & =S_{\Lambda}, \\
\mathcal{F}_{4} & =V_{\vec{\Lambda}},
\end{aligned}
$$

as expected. The counting of the physical degrees of freedom (dof) of the theory is as follows: 2 dof $=$ (Total number of canonical variables) - 2 (number of first-class constraints) - (number of second-class constraints), that is, dof $=2 N-5$. In the case of a gonihedric string immersed into a 4-dim Minkoswki spacetime, we have dof $=3$. Hence, there are three degrees of freedom for each normal vector of the worldsheet.

Under the Dirac bracket (55), the constraint algebra reads

$$
\begin{array}{ll}
\left\{\mathcal{S}_{\lambda}, \mathcal{S}_{\lambda^{\prime}}\right\}_{D}=0 & \left\{\mathcal{V}_{\vec{\lambda}}, S_{\Lambda}\right\}_{D}=\mathcal{S}_{\mathcal{L}_{\vec{\lambda}} \Lambda}-\mathcal{V}_{\vec{\lambda}_{1}}-V_{\vec{\Lambda}_{1}}, \\
\left\{\mathcal{S}_{\lambda}, \mathcal{V}_{\vec{\lambda}}\right\}_{D}=\mathcal{V}_{\overrightarrow{\lambda^{*}}} & \left\{\mathcal{V}_{\vec{\lambda}}, V_{\vec{\Lambda}}\right\}_{D}=\mathcal{V}_{[\vec{\lambda}, \vec{\Lambda}]}, \\
\left\{\mathcal{S}_{\lambda}, S_{\Lambda}\right\}_{D}=-\mathcal{S}_{\lambda_{1}}-S_{\Lambda_{1}} & \left\{S_{\Lambda}, S_{\Lambda^{\prime}}\right\}_{D}=\mathcal{S}_{\lambda_{2}}, \\
\left\{\mathcal{S}_{\lambda}, V_{\vec{\Lambda}}\right\}_{D}=-\mathcal{S}_{\mathcal{L}_{\vec{\Lambda}} \lambda} & \left\{S_{\Lambda}, V_{\vec{\Lambda}}\right\}_{D}=-S_{\mathcal{L}_{\vec{\Lambda}} \Lambda}+\mathcal{V}_{\vec{\lambda}_{2}}, \\
\left\{\mathcal{V}_{\vec{\lambda}}, \mathcal{V}_{\overrightarrow{\lambda^{\prime}}}\right\}_{D}=0 & \left\{V_{\vec{\Lambda}}, V_{\vec{\Lambda}^{\prime}}\right\}_{D}=V_{\left[\vec{\Lambda}, \overrightarrow{\Lambda^{\prime}}\right]},
\end{array}
$$


where, for the sake of simplicity, we have introduced the following definitions

$$
\begin{array}{ll}
\lambda^{* \sigma}=\lambda \lambda^{\sigma} & \Lambda_{1}^{\sigma}=\Lambda \lambda^{\sigma} \\
\lambda_{1}=\lambda \Lambda & \lambda_{2}=\left(N^{2} h^{\sigma \sigma}-N^{\sigma} N^{\sigma}\right)\left(\Lambda \mathcal{D}_{\sigma} \mathcal{D}_{\sigma} \Lambda^{\prime}-\Lambda^{\prime} \mathcal{D}_{\sigma} \mathcal{D}_{\sigma} \Lambda\right), \\
\Lambda_{1}=2 \Lambda \mathcal{L}_{\vec{N}} \lambda & \lambda_{2}^{\sigma}=\Lambda\left(N^{2} h^{\sigma \sigma}-N^{\sigma} N^{\sigma}\right) \mathcal{D}_{\sigma}^{2} \Lambda^{\sigma}, \\
\lambda_{1}^{\sigma}=2 \Lambda N^{\sigma} \mathcal{D}_{\sigma} \lambda^{\sigma} . &
\end{array}
$$

We would like to emphasize that the multiple appearance of the indices $\sigma$ does not indicate a tensor sum since we must recall that we have a unique spatial index. It is worthwhile to emphasize that this algebra has been computed on the first-class constraint surface where we have taken the second-class constraints as strong identities after introducing the Dirac bracket structure [26].

\subsection{Infinitesimal canonical transformations}

Regarding our symplectic analysis, for any classical observable $F \in \Gamma$, the Hamiltonian vector field

$$
X_{F}:=\int_{\Sigma_{t}} d \sigma\left(\frac{\delta F}{\delta p_{\mu}} \frac{\delta}{\delta X^{\mu}}+\frac{\delta F}{\delta P_{\mu}} \frac{\delta}{\delta \dot{X}^{\mu}}-\frac{\delta F}{\delta X^{\mu}} \frac{\delta}{\delta p_{\mu}}-\frac{\delta F}{\delta \dot{X} \mu} \frac{\delta}{\delta P_{\mu}}\right),
$$

generates a one-parameter family of canonical transformations $G \longrightarrow G+\delta_{F} G$, where $\delta_{F} G:=\epsilon\{G, F\}$, with $\epsilon$ being an infinitesimal quantity. In particular, the Hamiltonian vector fields associated with the first-class constraints (46), (47), (56) and (57), induce the infinitesimal canonical transformations

$$
\begin{aligned}
& X_{\mathcal{F}_{1}} \longrightarrow\left\{\begin{array} { l } 
{ \delta _ { \mathcal { S } _ { \lambda } } X ^ { \mu } = 0 , } \\
{ \delta _ { \mathcal { S } _ { \lambda } } \dot { X } ^ { \mu } = \epsilon _ { 1 } \lambda \dot { X } ^ { \mu } , } \\
{ \delta _ { \mathcal { S } _ { \lambda } } p _ { \mu } = 0 , } \\
{ \delta _ { \mathcal { S } _ { \lambda } } P _ { \mu } = - \epsilon _ { 1 } \lambda P _ { \mu } , }
\end{array} \quad X _ { \mathcal { F } _ { 3 } } \longrightarrow \left\{\begin{array}{l}
\delta_{S_{\Lambda}} X^{\mu}=\epsilon_{3} \Lambda \dot{X}^{\mu}, \\
\delta_{S_{\Lambda}} \dot{X}^{\mu}=-\epsilon_{3} \frac{\delta S_{\Lambda}}{\delta P_{\mu}}, \\
\delta_{S_{\Lambda}} p_{\mu}=-\epsilon_{3} \frac{\delta S_{\Lambda}}{\delta X^{\mu}} \\
\delta_{S_{\Lambda}} P_{\mu}=-\epsilon_{3} \frac{\delta S_{\Lambda}}{\delta \dot{X}^{\mu}}
\end{array}\right.\right. \\
& X_{\mathcal{F}_{2}} \longrightarrow\left\{\begin{array} { l } 
{ \delta _ { \mathcal { V } _ { \vec { \lambda } } } X ^ { \mu } = 0 , } \\
{ \delta _ { \mathcal { V } _ { \vec { \lambda } } } \dot { X } ^ { \mu } = \epsilon _ { 2 } \mathcal { L } _ { \vec { \lambda } } X ^ { \mu } , } \\
{ \delta _ { \mathcal { V } _ { \vec { \lambda } } } p _ { \mu } = \epsilon _ { 2 } \mathcal { L } _ { \vec { \lambda } } P _ { \mu } , } \\
{ \delta _ { \mathcal { V } _ { \vec { \lambda } } } P _ { \mu } = 0 , }
\end{array} \quad X _ { \mathcal { F } _ { 4 } } \longrightarrow \left\{\begin{array}{l}
\delta_{V_{\vec{\Lambda}}} X^{\mu}=\epsilon_{4} \mathcal{L}_{\vec{\Lambda}} X^{\mu}, \\
\delta_{V_{\vec{\Lambda}}} \dot{X}=\epsilon_{4} \mathcal{L}_{\vec{\Lambda}} \dot{X}^{\mu} \\
\delta_{V_{\vec{\Lambda}}} p_{\mu}=\epsilon_{4} \mathcal{L}_{\vec{\Lambda}} p_{\mu}, \\
\delta_{V_{\vec{\Lambda}}} P_{\mu}=\epsilon_{4} \mathcal{L}_{\vec{\Lambda}} P_{\mu}
\end{array}\right.\right.
\end{aligned}
$$

Note here that the $\epsilon_{i}(i=1, \ldots, 4)$ stands for an arbitrary gauge parameter corresponding to each of the first-class constraints $\mathcal{F}_{i}$, respectively. From (61), we may

infer that the constraint $V_{\vec{\Lambda}}$ is the generator of diffeomorphisms tangential to $\Sigma_{t}$, while $S_{\Lambda}$ is the generator of diffeomorphisms out of $\Sigma_{t}$ onto the worldsheet $m$. Analogously, $\mathcal{S}_{\lambda}$ is the generator of a parity transformation in the sub-sector of the phase space given by $\left\{\dot{X}^{\mu} ; P_{\mu}\right\}$, that is, the sector associated with the second-order in derivatives 
dependence in brane theories. Finally, we see that the vector constraint $\mathcal{V}_{\vec{\lambda}}$ only acts in the sub-sector $\left\{\dot{X}^{\mu} ; p_{\mu}\right\}$ by generating displacements in the orthogonal complement of this sub-sector, that is, in the sub-sector $\left\{X^{\mu} ; P_{\mu}\right\}$.

\section{Point-particle limit of the gonihedric string ac- tion}

The kinematic geometrical description we have performed on the gonihedric string model is based on the Gauss-Weingarten equations supplemented with integrability conditions [20]. In a similar manner, there is an analogue one-dimensional description for this framework, used for the description of relativistic curves in a background spacetime [16]. Bearing in mind the geometric construction of previous sections, we are able to obtain the point-like analogue for the gonihedric string mode $4^{4}$. Indeed, by considering the orthonormal basis $\left\{\dot{X}^{\mu}, n^{\mu}{ }_{i}\right\}$, related to the usual Frenet-Serret basis along the particle trajectory $C$, we are able to establish a straightforward correspondence with our formulation. This equivalence is compiled in Table 1. Note

\begin{tabular}{ccl}
\hline Higher-dimensional quantity & One-dimensional analogue & One-dimensional description \\
\hline$g_{a b}$ & $\gamma$ & Induced metric on the curve \\
$d^{2} x \sqrt{-g}$ & $d \xi \sqrt{-\gamma}$ & Element of line \\
$K_{a b}^{i}$ & $K^{i}$ & Extrinsic curvature along \\
$K^{i}$ & $k^{i}$ & the $i$-th normal \\
& & Mean extrinsic curvature along \\
& $\hat{k}^{i}=k^{i} / \sqrt{k^{j} k_{j}}$ & the $i$-th normal \\
$\widehat{K}^{i}=K^{i} / \sqrt{K^{j} K_{j}}$ & $\omega^{i j}$ & Unit vector \\
$\omega_{a}^{i j}$ & $\widetilde{\nabla}$ & Covarinsic twist derivative under \\
$\widetilde{\nabla}_{a}$ & & rotation of the normals
\end{tabular}

Table 1: Comparison among the higher-dimensional geometric quantities and the one-dimensional ones describing relativistic curves.

that in the one-dimensional case, the overdot stands for the derivative with respect to an arbitrary parameter, $\xi$, defined on $C$. In this framework, the so-called geodesic curvature $k$ given by $k=\sqrt{k^{i} k_{i}}$ is the one-dimensional analogue of the mean extrinsic curvature for extended objects. Following this line of reasoning, the action functional (1) reduces to the one-dimensional field theory

$$
S\left[X^{\mu}\right]=\beta \int_{C} d \xi \sqrt{-\gamma} k
$$

\footnotetext{
${ }^{4}$ In reference [27], the author considers a different perspective on the Hamiltonian construction of the point-particle limit described here by starting from a Lagrangian in its first order formalism.
} 
Here, the $X^{\mu}$ are describing the worldline $C$. The corresponding field equations, $\widetilde{\Delta} \hat{k}^{i}=0$, are directly obtained from (6), by using the terms given in Table 1 , and are in agreement with the results found in [28, 29, 30, 32, 31, 16]. The classical motion is confined on a plane such that the linear action in $k, 62$, turns out to be topological. On physical grounds, this action describes the motion of massless particles where the constant $\beta$ plays the role of the helicity of the particles.

The Hamiltonian framework for the action (62) may be directly obtained from the analysis developed in the previous sections. However, we must have in mind that for the one-dimensional case we must not consider any contribution coming from the quantities characterized by the spatial index $\sigma$. In particular, for the one-dimensional case it turns out that, besides the primary and secondary constraints given by (17), (19), 26) and (28), we have a tertiary constraint given by

$$
C_{5}:=p^{2}=0
$$

This extra constraint may be inferred from (103) by eliminating the spatial contributions. By applying consistency conditions one may find that there are no further constraints for the point-particle limit. It is likewise simple, from an OstrogradskiHamilton point of view, to see that we have a complete first-class constrained system for this theory. A word of caution is in order. While the main symmetry for the gonihedric model is the invariance under reparametrizations, for the relativistic particle field theory (62) there is, in addition, the presence of a $W$-symmetry which exhibits a deeper geometric structure. This last topic has been extensively addressed in reference [33].

\section{Concluding remarks}

In this paper we have developed the Hamiltonian formulation of the gonihedric string propagating in a Minkowski spacetime. Our phase space description includes the presence of four first-class constraints while two second-class constraints are present in the theory. We believe it is pertinent to mention that, in Ref. [6], Savvidy considered the gonihedric string model in two classically equivalent versions: firstly, a theory for which the independent field variables are the embedding functions, $X^{\mu}$, and, secondly, a theory in which both the embedding functions, $X^{\mu}$, and the induced worldsheet metric, $g_{a b}$, are the independent variables. In this paper, we have opted for the first version, the so-called model A in Savvidy's work, with the intention to keep the natural geometric structures associated with the worldsheet by means of the original field variables, that is, the embedding functions. Though the action describing the theory involves a square root Lagrangian that may be allegedly difficult to manipulate, we were able to introduce an Ostrogradski-Hamiltonian analysis for the 
constraint content of the model. In particular, bearing in mind the previous developed geometrical description for extended objects, we handled in an elementary manner both the highly non-linear and the second-order in derivative dependence of the model on the embedding functions. Thus, our approach allowed us to construct not only the canonical Hamiltonian but also to identify the complete set of constraints. After decomposing the set of constraints into first- and second-class, we introduced the Dirac bracket which enforces the second-class constraints as strong identities, and we were able to recognize the gauge symmetries of the theory by studying the infinitesimal canonical transformations generated by the first-class constraints. Also, as a consequence of the constraint characterization, we obtain the correct number of degrees of freedom for the gonihedric string model. Furthermore, as a byproduct of our geometrical formulation, we straightforwardly recover the Hamiltonian formulation for the point-particle limit of the gonihedric string theory by establishing a comparison between the higher-dimensional geometric quantities and the ones describing relativistic curves.

Even though a complete Hamiltonian construction for the gonihedric string theory may be analyzed by considering the Lagrangian in its first-order formalism, the analysis introduced here allowed us to take advantage of the well-established Hamiltonian description for extended objects. This last issue resulted completely convenient in order to characterize the constrained structure of the model. We also hope that the Hamiltonian formalism described here may pave the way for the quantum counterpart for the gonihedric string from a canonical perspective, as discussed for several extended objects when they are specialized to a particular geometry. Referring to this, despite that the abstract canonical quantization procedure is clear, when considering the embedding functions as the independent field variables and the highly non-linear dependence on the second-order derivatives of $X^{\mu}$ in the gonihedric string action, as similarly occurs in the Born-Infeld-type actions, the passage to the quantum theory is rather involved. Perhaps, to lighten the general problems of canonical quantization and to be able to obtain relevant physical information, it would be worth investigating only normal deformations. In such a case, the canonical constraints (17), (18), (19) and (27) do not undergo any change while (26) and (28) get huge simplification. We will have occasion to explore it in its full generality elsewhere. In this spirit, it will be interesting to try to connect the physical implications arising from our Hamiltonian development with the existing quantization approach which exploits a Weyl invariance present in the so-called model B [6, 7]. This connection is still a work in progress. 


\section{Acknowledgments}

ER thanks ProdeP-México, CA-UV-320: Álgebra, Geometría y Gravitación. Also, ER thanks the Departamento de Física de la Escuela Superior de Física y Matemáticas del Instituto Politécnico Nacional, México, where this work was developed during a sabbatical leave. AM would like to acknowledge financial support from ConacytMexico under project CB-2017-283838. This work was partially supported by Sistema Nacional de Investigadores, México.

\section{Appendix A. Poisson algebra of the constraints}

In this appendix we summarize the Poisson algebra among the constraint functions $\left\{\mathcal{S}_{\lambda}, \mathcal{V}_{\vec{\lambda}}, \mathcal{W}_{\phi}, S_{\Lambda}, V_{\vec{\Lambda}}, W_{\Phi}\right\}$. We proceed in an orderly way, avoiding repeated information by implicitly assuming the anticommutativity of the Poisson-brackets. Please note that the integrals denoted by $\mathbb{A}, \mathbb{B}, \mathbb{C}$ and $\mathbb{F}$ appearing below are explicitly given in the Subsection 7.1, where we have collected some useful formulas.

First, for the constraint $\mathcal{S}_{\lambda}$ we obtain

$$
\begin{array}{rlrl}
\left\{\mathcal{S}_{\lambda}, \mathcal{S}_{\lambda^{\prime}}\right\} & =0, & \\
\left\{\mathcal{S}_{\lambda}, \mathcal{V}_{\vec{\lambda}}\right\} & =\mathcal{V}_{\overrightarrow{\lambda^{\prime}}}, & \lambda^{\prime}=\lambda \lambda^{\sigma}, \\
\left\{\mathcal{S}_{\lambda}, \mathcal{W}_{\phi}\right\} & =0, & & \\
\left\{\mathcal{S}_{\lambda}, S_{\Lambda}\right\} & =-\mathcal{S}_{\lambda^{\prime}}-S_{\Lambda^{\prime}}, & \\
\left\{\mathcal{S}_{\lambda}, V_{\vec{\Lambda}}\right\} & =-\mathcal{S}_{\mathcal{L}_{\vec{\Lambda}} \lambda}, & \\
\left\{\mathcal{S}_{\lambda}, W_{\Phi}\right\} & =W_{\lambda \Phi} &
\end{array}
$$

Second, for the constraint $\mathcal{V}_{\vec{\lambda}}$ we get

$$
\begin{aligned}
\left\{\mathcal{V}_{\vec{\lambda}}, \mathcal{V}_{\overrightarrow{\lambda^{\prime}}}\right\} & =0 \\
\left\{\mathcal{V}_{\vec{\lambda}}, \mathcal{W}_{\phi}\right\} & =0 \\
\left\{\mathcal{V}_{\vec{\lambda}}, S_{\Lambda}\right\} & =\mathcal{S}_{\mathcal{L}_{\vec{\lambda}} \Lambda}-\mathcal{V}_{\overrightarrow{\lambda^{\prime}}}-V_{\vec{\Lambda}^{\prime}}, \quad \lambda^{\prime}=2 \Lambda N^{\sigma} \mathcal{D}_{\sigma} \lambda^{\sigma} \quad \Lambda^{\prime \sigma}=\Lambda \lambda^{\sigma} \\
\left\{\mathcal{V}_{\vec{\lambda}}, V_{\vec{\lambda}}\right\} & =\mathcal{V}_{[\vec{\lambda}, \vec{\Lambda}]}, \\
\left\{\mathcal{V}_{\vec{\lambda}}, W_{\Phi}\right\} & =0
\end{aligned}
$$

Third, for the constraint $\mathcal{W}_{\phi}$ the PB's read

$$
\begin{aligned}
\left\{\mathcal{W}_{\phi}, \mathcal{W}_{\phi^{\prime}}\right\} & =0 \\
\left\{\mathcal{W}_{\phi}, S_{\Lambda}\right\} & =\mathcal{S}_{\lambda^{*}}+\mathcal{V}_{\lambda^{*}}+\mathcal{W}_{\phi^{*}}-W_{\Phi^{*}}
\end{aligned}
$$




$$
\begin{aligned}
\left\{\mathcal{W}_{\phi}, V_{\vec{\Lambda}}\right\} & =-\mathcal{W}_{\mathcal{L}_{\hat{\Lambda}} \phi}, \\
\left\{\mathcal{W}_{\phi}, W_{\Phi}\right\} & =-\mathcal{S}_{\lambda^{\prime}}+\mathcal{V}_{\overrightarrow{\lambda^{\prime}}}+\mathcal{W}_{\phi^{\prime}}-S_{\Lambda^{\prime}}+V_{\overrightarrow{\Lambda^{\prime}}}+\mathbb{A}
\end{aligned}
$$

where

$$
\begin{aligned}
\lambda^{*} & =4 \phi \Lambda N^{2} h^{\sigma \sigma}\left(P \cdot \mathcal{D}_{\sigma} \mathcal{D}_{\sigma} X\right) \\
\lambda^{* \sigma} & =-4 \phi \Lambda N^{2} h^{\sigma \sigma}\left(P \cdot \mathcal{D}_{\sigma} \dot{X}\right) \\
\phi^{*} & =\phi \mathcal{L}_{\vec{N}} \Lambda-\Lambda \mathcal{L}_{\vec{N}} \phi+2 \phi \Lambda N k \\
\Phi^{*} & =2 \phi \Lambda N^{2} \\
\lambda^{\prime} & =2 \mathcal{L}_{\vec{N}}\left(\phi \Phi P^{2}\right) \\
\lambda^{\prime \sigma} & =2 \Phi\left(\mathcal{D}_{\sigma} \phi\right) N^{2} P^{2} h^{\sigma \sigma}-2 \phi\left(\mathcal{D}_{\sigma} \Phi\right)\left(N^{2} h^{\sigma \sigma}-N^{\sigma} N^{\sigma}\right) P^{2} \\
& +2 \Phi N^{\sigma} \mathcal{D}_{\sigma}\left(\phi P^{2} N^{\sigma}\right)-2 \phi \Phi N^{2} h^{\sigma \sigma}\left(P \cdot \mathcal{D}_{\sigma} P\right), \\
\phi^{\prime} & =2 \phi \Phi h^{\sigma \sigma}\left(P \cdot \mathcal{D}_{\sigma} \mathcal{D}_{\sigma} X\right), \\
\Lambda^{\prime} & =2 \phi \Phi P^{2} \\
\Lambda^{\prime} \sigma & =2 \phi \Phi N^{\sigma} P^{2} .
\end{aligned}
$$

Fourth, for the constraint $S_{\Lambda}$ we have

$$
\begin{aligned}
\left\{S_{\Lambda}, S_{\Lambda^{\prime}}\right\} & =\mathcal{S}_{\lambda^{\prime}} \quad \lambda^{\prime}=\left(N^{2} h^{\sigma \sigma}-N^{\sigma} N^{\sigma}\right)\left(\Lambda \mathcal{D}_{\sigma} \mathcal{D}_{\sigma} \Lambda^{\prime}-\Lambda^{\prime} \mathcal{D}_{\sigma} \mathcal{D}_{\sigma} \Lambda\right) \\
\left\{S_{\Lambda}, V_{\vec{\Lambda}}\right\} & =-S_{\mathcal{L}_{\vec{\Lambda}} \Lambda}+\mathcal{V}_{\overrightarrow{\lambda^{\prime}}}, \quad \lambda^{\prime \sigma}=\Lambda\left(N^{2} h^{\sigma \sigma}-N^{\sigma} N^{\sigma}\right) \mathcal{D}_{\sigma} \mathcal{D}_{\sigma} \Lambda^{\sigma} \\
\left\{S_{\Lambda}, W_{\Phi}\right\} & =\mathcal{S}_{\lambda^{\prime \prime}}+\mathcal{V}_{\vec{\lambda}^{\prime \prime}}+\mathcal{W}_{\phi^{\prime \prime}}-S_{\Lambda^{\prime \prime}}+V_{\bar{\Lambda}^{\prime \prime}}+W_{\Phi^{\prime \prime}}+\mathbb{B}
\end{aligned}
$$

where

$$
\begin{aligned}
\lambda^{\prime \prime} & =-2 \Lambda\left(\mathcal{L}_{\vec{N}} \Phi\right) h^{\sigma \sigma}\left(P \cdot \mathcal{D}_{\sigma} \mathcal{D}_{\sigma} X\right)-2 \Phi \mathcal{D}_{\sigma}\left[\Lambda h^{\sigma \sigma}\left(P \cdot \mathcal{D}_{\sigma} \dot{X}\right)\right] \\
\lambda^{\prime \prime \sigma} & =2 \Phi h^{\sigma \sigma} \mathcal{D}_{\sigma}\left[\Lambda\left(N^{2} h^{\sigma \sigma}-N^{\sigma} N^{\sigma}\right)\left(P \cdot \mathcal{D}_{\sigma} \mathcal{D}_{\sigma} X\right)\right] \\
& +2 \Phi h^{\sigma \sigma} \mathcal{D}_{\sigma}\left[\Lambda N^{\sigma}\left(P \cdot \mathcal{D}_{\sigma} \dot{X}\right)\right]+2 \Lambda\left(\mathcal{L}_{\vec{N}} \Phi\right) h^{\sigma \sigma}\left(P \cdot \mathcal{D}_{\sigma} \dot{X}\right) \\
\phi^{\prime \prime} & =\Lambda\left(h^{\sigma \sigma}-\frac{N^{\sigma} N^{\sigma}}{N^{2}}\right) \mathcal{D}_{\sigma} \mathcal{D}_{\sigma} \Phi \\
\Lambda^{\prime \prime} & =2 \Lambda \Phi h^{\sigma}\left(P \cdot \mathcal{D}_{\sigma} \mathcal{D}_{\sigma} X\right) \\
\Lambda^{\prime \prime} \sigma & =2 \Lambda \Phi h^{\sigma \sigma}\left(P \cdot \mathcal{D}_{\sigma} \dot{X}\right) \\
\Phi^{\prime \prime} & =\Lambda \mathcal{L}_{\vec{N}} \Phi-2 \Phi \mathcal{D}_{\sigma}\left(\Lambda N^{\sigma}\right)
\end{aligned}
$$

Fifth, for the constraint $V_{\vec{\Lambda}}$ we obtain

$$
\begin{aligned}
\left\{V_{\vec{\Lambda}}, V_{\overrightarrow{\Lambda^{\prime}}}\right\} & =V_{\left[\vec{\Lambda}, \overrightarrow{\Lambda^{\prime}}\right]}, \\
\left\{V_{\vec{\Lambda}}, W_{\Phi}\right\} & =W_{\mathcal{L}_{\vec{\Lambda}} \Phi}+\mathbb{C} .
\end{aligned}
$$


Sixth, for the constraint $W_{\Phi}$ we only need to calculate the PB

$$
\left\{W_{\Phi}, W_{\Phi^{\prime}}\right\}=\mathcal{S}_{\lambda^{\prime}}+\mathcal{V}_{\overrightarrow{\lambda^{\prime}}}-V_{\overrightarrow{\Lambda^{\prime}}}+\mathbb{F}
$$

where,

$$
\begin{aligned}
\lambda^{\prime} & =h^{\sigma \sigma}\left[\Phi \mathcal{D}_{\sigma}\left(P \cdot \mathcal{D}_{\sigma}\left(\Phi^{\prime} P\right)\right)-\Phi^{\prime} \mathcal{D}_{\sigma}\left(P \cdot \mathcal{D}_{\sigma}(\Phi P)\right)\right] \\
\lambda^{\prime \sigma} & =h^{\sigma \sigma}\left\{2 \Phi^{\prime} \mathcal{D}^{\sigma}\left[P \cdot \mathcal{D}_{(\sigma}(\Phi P) N_{\sigma)}\right]+\left(\mathcal{L}_{\vec{N}} \Phi^{\prime}\right)\left[P \cdot \mathcal{D}_{\sigma}(\Phi P)\right]\right. \\
& \left.-2 \Phi \mathcal{D}^{\sigma}\left[P \cdot \mathcal{D}_{(\sigma}\left(\Phi^{\prime} P\right) N_{\sigma)}\right]-\left(\mathcal{L}_{\vec{N}} \Phi\right)\left[P \cdot \mathcal{D}_{\sigma}\left(\Phi^{\prime} P\right)\right]\right\} \\
\Lambda^{\prime} \sigma & =h^{\sigma \sigma}\left(\Phi \partial_{\sigma} \Phi^{\prime}-\Phi^{\prime} \partial_{\sigma} \Phi\right) P^{2} .
\end{aligned}
$$

\subsection{Useful formulae}

In this subsection, we include some useful formulas that include relevant information

on either the PB's described above or the Hamiltonian field equations of Section 3.1.

In the first instance, we consider the integrals in the previous subsection which are explicitly given by

$$
\begin{aligned}
\mathbb{A} & =\int_{\Sigma_{t}} 4 \phi \Phi \alpha_{p}^{2} h h^{\sigma \sigma}\left(P \cdot \mathcal{D}_{\sigma} \mathcal{D}_{\sigma} X\right) \\
\mathbb{B} & =\int_{\Sigma_{t}} \Lambda \Phi\left(p^{2}-2 N^{\sigma} p \cdot \mathcal{D}_{\sigma} P\right)+\alpha_{p}^{2} h\left(h^{\sigma \sigma}-\frac{N^{\sigma} N^{\sigma}}{N^{2}}\right) \Lambda \mathcal{D}_{\sigma} \mathcal{D}_{\sigma} \Phi \\
& +4 \Lambda \Phi\left(N^{2} h^{\sigma \sigma}-N^{\sigma} N^{\sigma}\right) h^{\sigma \sigma}\left(P \cdot \mathcal{D}_{\sigma} \mathcal{D}_{\sigma} X\right)\left(P \cdot \mathcal{D}_{\sigma} \mathcal{D}_{\sigma} X\right) \\
& +8 \Lambda \Phi h^{\sigma \sigma} N^{\sigma}\left(P \cdot \mathcal{D}_{\sigma} \mathcal{D}_{\sigma} X\right)\left(P \cdot \mathcal{D}_{\sigma} \dot{X}\right) \\
& -4 \Lambda \Phi h^{\sigma \sigma}\left(P \cdot \mathcal{D}_{\sigma} \dot{X}\right)\left(P \cdot \mathcal{D}_{\sigma} \dot{X}\right)+\Lambda \Phi\left(N^{2} h^{\sigma \sigma}-N^{\sigma} N^{\sigma}\right)\left(P \cdot \mathcal{D}_{\sigma} \mathcal{D}_{\sigma} P\right) \\
& +2 \Lambda\left(N^{2} h^{\sigma \sigma}-N^{\sigma} N^{\sigma}\right) \mathcal{D}_{\sigma} \Phi\left(P \cdot \mathcal{D}_{\sigma} P\right)+N^{2} h^{\sigma \sigma} \mathcal{D}_{\sigma} \Lambda\left(P \cdot \mathcal{D}_{\sigma}(\Phi P)\right) \\
& -\Lambda N^{\sigma} \mathcal{D}_{\sigma} N^{\sigma}\left(P \cdot \mathcal{D}_{\sigma}(\Phi P)\right)+\Lambda N h^{\sigma \sigma} \mathcal{D}_{\sigma} N\left(P \cdot \mathcal{D}_{\sigma}(\Phi P)\right) \\
& +N^{\sigma} N^{\sigma} \mathcal{D}_{\sigma} \Lambda\left(P \cdot \mathcal{D}_{\sigma}(\Phi P)\right)+\Lambda\left(\mathcal{L}_{\vec{N}} \Phi\right)\left(P \cdot \mathcal{D}_{\sigma}\left(N^{\sigma} P\right)\right) \\
- & 2 \Phi \mathcal{D}_{\sigma}\left(\Lambda N^{\sigma}\right)\left(P \cdot \mathcal{D}_{\sigma}\left(N^{\sigma} P\right)\right)-2 \Lambda\left(\mathcal{L}_{\vec{N}} \Phi\right) N^{\sigma}\left(P \cdot \mathcal{D}_{\sigma} P\right) \\
- & 2\left(\mathcal{L}_{\vec{N}} \Phi\right) \mathcal{D}_{\sigma}\left(\Lambda N^{\sigma}\right) P^{2}, \\
\mathbb{C}= & \int_{\Sigma_{t}}\left[N^{\sigma}\left(\mathcal{L}_{\vec{\Lambda}} \Phi\right)-\Lambda^{\sigma}\left(\mathcal{L}_{\vec{N}} \Phi\right)-\Phi\left(\mathcal{L}_{\vec{\Lambda}} N^{\sigma}\right)\right]\left(P \cdot \mathcal{D}_{\sigma} P\right) \\
+ & {\left[\left(\mathcal{D}_{\sigma} N^{\sigma}\right)\left(\mathcal{L}_{\vec{\Lambda}} \Phi\right)-\left(\mathcal{D}_{\sigma} \Lambda^{\sigma}\right)\left(\mathcal{L}_{\vec{N}} \Phi\right)-\left(\mathcal{D}_{\sigma} \Phi\right)\left(\mathcal{L}_{\vec{\Lambda}} N^{\sigma}\right)\right] P^{2}, } \\
\mathbb{F}= & \int_{\Sigma_{t}} 2 h^{\sigma \sigma}\left(\Phi \partial_{\sigma} \Phi^{\prime}-\Phi^{\prime} \partial_{\sigma} \phi\right)\left[P \cdot \mathcal{D}_{\sigma} \dot{X}-N^{C}\left(P \cdot \mathcal{D}_{\sigma} \mathcal{D}_{\sigma} X\right)\right] P^{2} .
\end{aligned}
$$

Note that this integrals are fundamental in the definition of the Dirac bracket in Section 4, and also to understand the emergence of the tertiary constraint (63) in section 5 . 
In the second instance, in order to reproduce the Hamiltonian field equations of Subsection 3.1 we keep in mind the general expression for the acceleration of an extended object $\Sigma$ is [14]

$$
\begin{aligned}
\ddot{X}^{\mu} & =\left(\dot{N}_{A}+N \mathcal{D}_{A} N-N^{B} \mathcal{D}_{A} N_{B}\right) \epsilon^{\mu A}+\left(\dot{N}+N^{A} \mathcal{D}_{A} N+N^{A} N^{B} k_{A B}\right) \eta^{\mu} \\
& +\left(n^{i} \cdot \ddot{X}\right) n^{\mu}{ }_{i}
\end{aligned}
$$

together with the important formulae for the temporal derivatives of the induced metric and its determinant,

$$
\begin{aligned}
\dot{h}_{A B} & =2 N k_{A B}+2 \mathcal{D}_{(A} N_{B)}, \\
\dot{h} & =2 h\left(N h^{A B} k_{A B}+\mathcal{D}_{A} N^{A}\right),
\end{aligned}
$$

respectively, where $A, B=1,2, \ldots, p$; here, $p$ is the dimension of the extended object.

\section{Appendix B. Functional derivatives of the con- straints}

In this Appendix, we collect the functional derivatives of the constraints which are relevant not only for the PB's described in Section 7 but also for the construction of the infinitesimal canonical transformations of Subsection 4.1. As before, we proceed in an orderly way, by considering in the first step the primary constraints $\mathcal{S}_{\lambda}, \mathcal{V}_{\vec{\lambda}}, \mathcal{W}_{\phi}$, while in the following steps we consider the secondary constraints $S_{\Lambda}, V_{\vec{\Lambda}}$ and $W_{\Phi}$, respectively.

First,

$$
\begin{array}{ll}
\frac{\delta \mathcal{S}_{\lambda}}{\delta \dot{X}^{\mu}}=\lambda P_{\mu} & \frac{\delta \mathcal{S}_{\lambda}}{\delta P_{\mu}}=\lambda \dot{X}^{\mu}, \\
\frac{\delta \mathcal{V}_{\vec{\lambda}}}{\delta X^{\mu}}=-\partial_{\sigma}\left(\lambda^{\sigma} P_{\mu}\right)=-\mathcal{L}_{\vec{\lambda}} P_{\mu} & \frac{\delta \mathcal{V}_{\vec{\lambda}}}{\delta P_{\mu}}=\lambda^{\sigma} \partial_{\sigma} X^{\mu}=\mathcal{L}_{\vec{\lambda}} X^{\mu} \\
\frac{\delta \mathcal{W}_{\phi}}{\delta \dot{X}^{\mu}}=-2 \phi N P^{2} \eta_{\mu} & \frac{\delta \mathcal{W}_{\phi}}{\delta P_{\mu}}=2 \phi N^{2} P^{\mu}, \\
\frac{\delta \mathcal{W}_{\phi}}{\delta X^{\mu}}=-\partial_{\sigma}\left(2 \phi N N^{\sigma} P^{2} \eta_{\mu}\right)+\partial_{\sigma}\left(2 \alpha \phi h h^{\sigma \sigma} \partial_{\sigma} X_{\mu}\right),
\end{array}
$$

where $\lambda$ is a test field of weight 0 and $\phi$ is a test field of weight -1 .

Second,

$$
\frac{\delta S_{\Lambda}}{\delta P_{\mu}}=\Lambda\left(N^{2} h^{\sigma \sigma}-N^{\sigma} N^{\sigma}\right) \mathcal{D}_{\sigma} \mathcal{D}_{\sigma} X^{\mu}+2 \Lambda N^{\sigma} \mathcal{D}_{\sigma} \dot{X}^{\mu} \quad \frac{\delta S_{\Lambda}}{\delta p_{\mu}}=\Lambda \dot{X}^{\mu}
$$




$$
\begin{aligned}
\frac{\delta S_{\Lambda}}{\delta \dot{X}^{\mu}} & =\Lambda p_{\mu}-2 \Lambda N h^{\sigma \sigma}\left(P \cdot \mathcal{D}_{\sigma} \mathcal{D}_{\sigma} X\right) \eta_{\mu}-2 \Lambda N^{\sigma}\left(P \cdot \mathcal{D}_{\sigma} \mathcal{D}_{\sigma} X\right) \epsilon_{\mu}{ }^{\sigma} \\
& +2 \Lambda\left(P \cdot \mathcal{D}_{\sigma} \dot{X}\right) \epsilon_{\mu}{ }^{\sigma}-\mathcal{D}_{\sigma}\left(2 \Lambda N^{\sigma} P_{\mu}\right) \\
\frac{\delta S_{\Lambda}}{\delta X^{\mu}} & =-\mathcal{D}_{\sigma}\left[2 \Lambda N h^{\sigma \sigma} N^{\sigma}\left(P \cdot \mathcal{D}_{\sigma} \mathcal{D}_{\sigma} X\right) \eta_{\mu}\right]+\mathcal{D}^{\sigma}\left[2 \Lambda N^{\sigma}\left(P \cdot \mathcal{D}_{\sigma} \mathcal{D}_{\sigma} X\right) \dot{X}_{\mu}\right] \\
& +\mathcal{D}_{\sigma}\left[2 \Lambda N^{2} h^{\sigma \sigma} h^{\sigma \sigma}\left(P \cdot \mathcal{D}_{\sigma} \mathcal{D}_{\sigma} X\right) \epsilon_{\mu \sigma}\right]-\mathcal{D}^{\sigma}\left[2 \Lambda\left(P \cdot \mathcal{D}_{\sigma} \dot{X}\right) \dot{X}_{\mu}\right] \\
& -\mathcal{D}^{\sigma}\left[4 \Lambda N^{\sigma}\left(P \cdot \mathcal{D}_{\sigma} \mathcal{D}_{\sigma} X\right) N_{\sigma} \epsilon_{\mu}{ }^{\sigma}\right]+\mathcal{D}^{\sigma}\left[4 \Lambda\left(P \cdot \mathcal{D}_{\sigma} \dot{X}\right) N_{\sigma} \epsilon_{\mu}{ }^{\sigma}\right] \\
& +\mathcal{D}_{\sigma} \mathcal{D}_{\sigma}\left[\Lambda\left(N^{2} h^{\sigma \sigma}-N^{\sigma} N^{\sigma}\right) P_{\mu}\right]
\end{aligned}
$$

where $\Lambda$ is test field of weight 0 .

Third,

$$
\begin{array}{ll}
\frac{\delta V_{\vec{\Lambda}}}{\delta \dot{X}^{\mu}}=-\partial_{\sigma}\left(\Lambda^{\sigma} P_{\mu}\right)=-\mathcal{L}_{\vec{\Lambda}} P_{\mu} & \frac{\delta V_{\vec{\Lambda}}}{\delta X^{\mu}}=-\partial_{\sigma}\left(\Lambda^{\sigma} p_{\mu}\right)=-\mathcal{L}_{\vec{\Lambda}} p_{\mu} \\
\frac{\delta V_{\vec{\Lambda}}}{\delta P_{\mu}}=\Lambda^{\sigma} \partial_{\sigma} \dot{X}^{\mu}=\mathcal{L}_{\vec{\Lambda}} \dot{X}^{\mu} & \frac{\delta V_{\vec{\Lambda}}}{\delta p_{\mu}}=\Lambda^{\sigma} \partial_{\sigma} X^{\mu}=\mathcal{L}_{\vec{\Lambda}} X^{\mu}
\end{array}
$$

Fourth,

$$
\begin{aligned}
\frac{\delta W_{\Phi}}{\delta X^{\mu}} & =-\mathcal{D}^{\sigma}\left[P \cdot \mathcal{D}_{\sigma}(\Phi P) \dot{X}_{\mu}\right]+\mathcal{D}_{\sigma}\left[2 P \cdot \mathcal{D}^{\sigma}(\Phi P) N^{\sigma} \epsilon_{\mu \sigma}\right] \\
\frac{\delta W_{\Phi}}{\delta \dot{X}^{\mu}} & =P \cdot \mathcal{D}_{\sigma}(\Phi P) \epsilon_{\mu}{ }^{\sigma} \\
\frac{\delta W_{\Phi}}{\delta p_{\mu}} & =\Phi P^{\mu} \\
\frac{\delta W_{\Phi}}{\delta P_{\mu}} & =\Phi p^{\mu}+\left(N^{\sigma} \mathcal{D}_{\sigma} \Phi-\Phi \mathcal{D}_{\sigma} N^{\sigma}\right) P^{\mu}=\Phi p^{\mu}+\left(\mathcal{L}_{\vec{N}} \Phi\right) P^{\mu}
\end{aligned}
$$

where $\Phi$ is a test field of weight -1 .

\section{References}

[1] R. V. Ambartzumanian, G. K. Savvidy, K. G. Savvidy and G. S. Sukiasian, Phys. Lett. B275, 99-102 (1992).

[2] G. K. Savvidy and K. G. Savvidy, Mod. Phys. Lett. A8, 2963-2972 (1992), arXiv:hep-th/9301001.

[3] G. K. Savvidy and K. G. Savvidy, Int. J. Mod. Phys. A8, 3993-4011 (1993), arXiv:hep-th/9208041. 
[4] G. K. Savvidy and R. Manvelyan, Phys. Lett. B533, 138-145 (2002), arXiv:hep-th/0111203.

[5] A. R. Fazio and G. K. Savvidy, Mod. Phys. Lett. A18, 2817-2828 (2003), arXiv:hep-th/0307267.

[6] G. K. Savvidy, Phys. Lett. B552, 72-80 (2003).

[7] G. K. Savvidy, Int. J. Mod. Phys. A19, 3171-3194 (2004), arXiv:hep-th/0310085.

[8] G. K. Savvidy and F. J. Wegner, Nucl. Phys. B413, 605-613 (1994), arXiv:hep-th/9308094.

[9] G. K. Savvidy, K. G. Savvidy and F. J. Wegner, Nucl. Phys. B443, 565-580 (1995), arXiv:hep-th/9503213.

[10] P. Dimopoulos, D. Espriu, E. Jané and A. Prats, Phys. Rev. E 66, 056112 (2002), arXiv: cond-mat/0204403

[11] G. Savvidy, Mod. Phys. Lett. B29, 1550203 (2015), arXiv:1501.01394 [hep-th].

[12] G. Savvidy, Gravity with linear action and gravitational singularities, In Proceedings of the 17th Hellenic School and Workshops on Elementary Particle Physics and Gravity (CORFU2017), 318, 171 (2018), 1705.01459 [hep-th].

[13] E. Rojas, Int. J. Mod. Phys. A32, 1750192 (2017), arXiv:1711.01019v2 [hep-th].

[14] R. Capovilla, J. Guven and E. Rojas, Class. Quantum Grav. 21, 5563-5585 (2004), arXiv:hep-th/0404178.

[15] B. Carter, Brane dynamics for treatment of cosmic strings and vortons, in "Recent Developments in Gravitation and Mathematics", Proc. 2nd Mexican School on Gravitation and Mathematical Physics, eds. A. Garcia, C. Lammerzahl, A. Macias, T. Matos and D. Nuñez. (Science Network Publishing, Konstanz, 1997); arXiv:hep-th/9705172.

[16] G. Arreaga, R. Capovilla and J. Guven, Ann. Phys. 279, 126-158 (2000), arXiv:hep-th/0002088.

[17] B. Y. Cheng, Geometry of Submanifolds (Dekker, 1973).

[18] M. Dajczer, Submanifolds and Isometric Immersions (Publish or Perish, 1990).

[19] J. Guven, Phys. Rev. D48, 4604-4608 (1993), arXiv:gr-qc/9304032. 
[20] R. Capovilla and J. Guven, Phys. Rev. D51, 6736-6743 (1995), arXiv:gr-qc/9411060v2.

[21] S. Kobayashi and K. Nomizu, Foundations of Differential Geometry: Volume II (Interscience, New York, 1969).

[22] R. Capovilla, J. Guven and E. Rojas, Nucl. Phys. B Proc. Suppl. 88,337-340 (2000), arXiv:hep-th/0004031.

[23] R. M. Wald, General Relativity (University of Chicago Press, 1984).

[24] V. V. Nesterenko, J. Phys. A: Math. Gen. 22, 1673-1687 (1989).

[25] P. A. M. Dirac, Proc. Roy. Soc. A 246333343 (1958)

[26] M. Henneaux and C. Teitelboim, Quantization of Gauge Systems (Princeton University Press, 1994).

[27] A. Nichols, Acta Phys. Pol. B34, 5009-5020 (2003).

[28] M. S. Plyuschay, Mod. Phys. Lett. A4, 837 (1988).

[29] M. S. Plyuschay, Int. J. Mod. Phys. A4, 3851 (1989).

[30] M. S. Plyuschay, Phys. Lett. B243, 383 (1990).

[31] V. V. Nesterenko, A. Feoli and G. Scarpetta, Class. Quantum Grav. 13, 12011211 (1996), arXiv:hep-th/9505064.

[32] D. Zoller, Phys. Rev. Lett. 65, 2236 (1990).

[33] E. Ramos and J. Roca, Nucl. Phys. B436, 529-541 (1995), arXiv:hep-th/9408019. 